Voix et Images

volxetimages

\title{
Les enfants du déclin
}

\section{Jacques Michon}

Volume 12, numéro 2 (35), hiver 1987

Jacques Brault

URI : https://id.erudit.org/iderudit/200643ar

DOI : https://doi.org/10.7202/200643ar

Aller au sommaire du numéro

Éditeur(s)

Université du Québec à Montréal

ISSN

0318-9201 (imprimé)

1705-933X (numérique)

Découvrir la revue

Citer cet article

Michon, J. (1987). Les enfants du déclin. Voix et Images, 12(2), 331-333.

https://doi.org/10.7202/200643ar

Ce document est protégé par la loi sur le droit d'auteur. L'utilisation des services d'Érudit (y compris la reproduction) est assujettie à sa politique d'utilisation que vous pouvez consulter en ligne.

https://apropos.erudit.org/fr/usagers/politique-dutilisation/
Cet article est diffusé et préservé par Érudit.

Érudit est un consortium interuniversitaire sans but lucratif composé de l’Université de Montréal, l'Université Laval et l'Université du Québec à Montréal. Il a pour mission la promotion et la valorisation de la recherche. https://www.erudit.org/fr/ 


\section{Récit}

\section{Les enfants du déclin}

\section{par Jacques Michon, Université de Sherbrooke}

Chaque fin de siècle apporte ses discours annonciateurs de décadence. Le dernier film de Denys Arcand. le Déclin de l'empire américain, est venu illustrer avec humour et ironie ce sentiment d'une collectivité qui s'épuise, qui a perdu le sens de l'histoire, qui se dissout dans l'oisiveté, se décompose et se fractionne dans les plaisirs multiples de la société d'abondance. Chez Arcand, cette déchéance se confond avec celle d'une génération qui ne croit plus aux dieux qu'elle a adorés. L'échec d'une jeunesse qui avait l'impression de pouvoir changer le monde en 1968 se découvre plus hédoniste que révolutionnaire.

L'effondrement des idéologies communautaires, la crise des valeurs et des solutions collectives, la déprime post-référendaire ont favorisé la représentation d'individualités fortes et conquérantes dans la littérature romanesque récente. Le Florent Boissonneault du Matou représente bien ce type de héros volontaire, rusé, indépendant qui sait manipuler ses semblables et les soumettre à ses propres fins. Aucune morale collective ne détermine son action et ne vient entraver sa volonté d'affirmation personnelle, aucune.valeur autre que celle de la réussite sociale et de la préservation de la vie privée.

Le dernier roman de Jean Forest, l'Aube de Suse', met en scène un représentant de cette nouvelle génération. Au premier abord, le propos du récit rappelle celui de l'Été Rebecca de René Lapierre: un professeur d'université s'éprend d'une étudiante avec laquelle il a une liaison passionnée. Contrairement au personnage de Lapierre toutefois, celui de Forest ira jusqu'au bout d'une expérience qui sera le moteur d'une métamorphose. II quitte femme et enfant pour former un nouveau ménage fondé sur d'autres valeurs, celles de la révolution sexuelle, des drogues douces et du retour à la nature qui ont marqué les années 70 . L'aube de Suse, c'est l'aube des amants qui annonce un nouveau jour, mais aussi la fin de l'emprise matriarcale et matrimoniale. Pour Christian le monde ne semble plus exister que pour cette expérience unique du corps passionné. Narrateur de son histoire. il nous livre par le détail les étapes des entreprises et des ruses déployées pour faire la conquête de Suse. Toutes les métaphores guerrières de la tradition courtoise sont mobilisées pour exprimer les manœuvres de séduction du Chevalier d'amore.

Comme tous les professeurs fictifs, de Louis Marin (Bessette) à Léonard Troy (Lapierre), Christian se moque de lui-même et mêle l'ironie à ses entreprises conquérantes, il prend ses distances avec les images qu'il projette. Il est trop habitué à l'analyse et à l'introspection pour se prendre au jeu de la confession naïve ou se laisser enfermer dans un seul rôle. En fait il veut occuper toutes les positions. Les fantasmes du courtisan libidineux nous révèlent un personnage complexe qui, aux vertus du mâle entreprenant, mêle les attributs d'une féminité dévorante, pourvoyeuse, maternelle... Ainsi va-t-il 
jusqu'à parler de ses couilles mamalles et de ses règles; il s'agit de cracher à la face du monde que JE SUIS UNE MEERE!, écrit-il (voir Julien Bigras). Christian assume tous les rôles et ne laisse plus beaucoup de place à l'autre qui se trouve assujetti par cette passion unique et absolue. $\lambda$ la fin du récit. après une scène de jalousie violente et incontrôlable. il reprend ses esprits et entend un démon moqueur ricaner derrière son dos. Il découvre que malgré tout le déploiement de force, de séduction et de violence pour encerrcler et contrôler Suze, elle lui échappe.

On peut évoquer ici la fin du Matou où, croyant contrôler la situation et en avoir fini avec l'affreux Ratablavasky, Florent s'aperçoit que ce double démoniaque n'est pas disparu, qu'il est toujours là, irréductible, invaincu, prêt à le précipiter dans de nouvelles aventures et d'autres métamorphoses. lci comme là, le retour du refoulé, la revanche du double, la partie sombre et inconsciente du sujet refait sans cesse surface, entame son intégrité et annonce son déclin. Le triomphe d'ailleurs ne peut être éprouvé dans toute sa plénitude que sur fond de mort et de destruction. Que la menace fasse partie du programme narratif du héros (le double de Christian) ou qu'elle soit projetée dans la figure mythique du persécuteur (Ratablavasky). elle repose sur la même structure.

Le Récif du Prince de Jacques Savoie ${ }^{2}$, montre l'envers de la situation précédente, en plaçant le lecteur du côté de l'opprimé, du refoulé, de celui ou de celle qui subit la pression, la passion dominante et narcissique du sujet conquérant. Vassilie. 17 ans, est une enfant qui essaie de se faire une place entre une mère absente qu'elle ne voit qu'à la télévision et un père vedette d'émission pour enfants. L'écran cathodique constitue le medium. l'espace essentiel des rapports familiaux. Les parents sont des figures publiques et font partie de cette génération trop occupée d'elle-même pour se laisser distraire par sa progéniture. Tania écrit à son mari à propos de leurs deux filles:

Il m'arrive de croire que nous avons tout fait sauf les élever. Qu'elles sont aujourd'hui une histoire qu'on se raconte, une histoire modifiable à volonté selon qu'on aime ou qu'on aime pas la fin. Quand je ferme les yeux, je les confonds parfois. Je ne sais plus laquelle est laquelle. Je les imagine toutes deux assises devant l'écran de télévision à nous regarder faire notre numéro.

En effet les enfants sont transformés en spectateurs passifs des problèmes et des obsessions de leurs parents. Comment échapper à cette neutralisation médiatique? Pour rompre cette relation qui se confond avec la rumeur publique, Vassilie rêve de se retrouver seule, gardienne de phare sur un récif «en plein milieu du Saint-Laurent ". Le "récif du Prince" s'avérera finalement un espace imaginaire, un lieu transitionnel qui sera échangé pour un prince charmant qu'elle ira rejoindre à Paris et que sa mère n'avait pas su reconnaître. Car dans sa quête d'identité et d'authenticité la jeune fille découvre le manque, la faille dans le système de valeurs des parents par où elle pourra s'échapper. 
Après ce conte tout simple sur l'éducation sentimentale d'une jeune fille qui ne perd jamais le sens de l'humour, ni son sens critique, on pourra lire la Fin des jeux ${ }^{3}$ de Michel Gosselin qui explore, lui aussi, les rapports problématiques de l'enfant et de l'adulte. En attendant le retour de son père en vacances aux Antilles, Simon, 10 ans, s'enferme dans l'appartement de ce dernier pour écrire un conte et refaire le monde à sa manière. Inquiets de sa disparition, sa mère et son deuxième père lancent une enquête policière pour le retrouver. Encore ici la télévision va jouer un rôle important comme substitut du réel. C'est par elle que l'enfant voit ce qui se passe à l'extérieur de son refuge, qu'il est témoin des recherches dont il est l'objet. Pendant ce temps, Étienne, le père, rumine des idées sombres sous le soleil des tropiques. Il sait depuis peu qu'il est atteint d'une maladie mortelle, ses jours sont comptés, il n'arrive plus à trouver une raison de vivre. Ainsi le lecteur suit en alternance ces cheminements parallèles de l'enfant et de l'adulte qui finiront par se rencontrer l'instant d'un appel téléphonique.

Le discours du petit Simon constitue sans doute la réussite de ce court récit où l'auteur parvient à traduire le monde et le langage de l'enfant de dix ans. Quelques naïvetés linguistiques, qui traduisent l'idiolecte du jeune garçon, sembleront superflues (pédérastre pour pédiatre, circonscrit pour circoncis) dans la mesure où c'est moins le lexique qui évoque la vision de l'enfance que la syntaxe et le point de vue. Cette réussite m'apparaît comme une promesse. une étape avant l'œuvre. qui la prépare et l'annonce. La disparition du père. dont la présence est moins affirmée, représente peut-être une phase nécessaire. une dette à liquider avant de passer à autre chose. Le prochain épisode annoncé à la fin du livre nous le dira.

Contrairement aux enfants révoltés d'un Rẻjean Ducharme ou, dernièrement, d'un Daniel Gagnon ${ }^{4}$ qui cherchent à transgresser l'ordre établi et s'inscrivent dans la subversion. la déviance, la marginalité, les enfants de Savoie et Gosselin cherchent plutôt à se conformer aux modèles reçus. Vassilie suit les traces de sa mère en allant rejoindre son ex-amant à Paris, Simon écrit comme ses parents (son père écrit des pièces pour la radio, sa mère est romancière, son père adoptif compose des chansons). Ayant reçu la désorganisation familiale en héritage. ils recherchent, contrairement à leurs prédécesseurs, à retrouver un ordre perdu. Comme ils n'ont pas connu les règles rigides et structurantes que leurs parents se sont employé à détruire, ils ne connaissent pas la révolte, du moins pas au sens où on l'entend habituellement. En fait, les aînés leur ont confisqué cette possibilité en n'agissant pas comme des parents, c'est-à-dire comme des représentants de la loi et de l'ordre. En refusant d'être de "vrais" parents, les ainés se sont ainsi mis à l'abri de la contestation qu'ils ont eux-mêmes fomentée contre leurs prédécesseurs. Le refus de l'image parentale, prolongée jusque dans l'âge adulte, a eu pour effet d'engendrer des êtres préoccupés par l'idée de refaire le monde à l'image de "l'ancien».

Les visions de l'enfance qui nous sont transmises ici, ont plusieurs traits communs, dont celui de concevoir l'enfant comme un spectateur et un juge 
qui tient vis-à-vis de l'adulte la position de la raison et de la sagesse commune. Je ne peux manquer d'évoquer l'épisode pathétique de la fille de Christian qui, dans l'Aube de Suse, pour protester contre son père qui la néglige depuis sa liaison, va déféquer partout l'obligeant à s'occuper d'elle. La régression devient ainsj le langage d'une protestation qui ne peut se dire autrement; lorsque les parents n'agissent plus en parents, on les rappelle à l'ordre pour les enjoindre de jouer leur rôle comme il taut. Cette petite fable psychanalytique me semble résumer à elle seule la situation de ce retour à l'ordre, refusé par les tenants de la révolution familiale. En donnant la parole à ces nouveaux enfants, le romancier se donne ici la distance critique nécessaire pour montrer les limites et les paradoxes engendrés par les ruptures et les utopies de sa propre génération.

1. L'A ube de Suse, Montréal, Quinze, 1985, 203 pages, constitue le deuxième volet d'une trilogie intitulée "La Mère. le Fils et le Saint-Esprit". Le premier tome, qui raconte l'adolescence du protagoniste, a été publié en 1984 sous le titre de Nourrice!... Nourrice!.... Montréal. Quinze, $185 \mathrm{p}$.

2. Le Récif du prince, Montréal. Boréal Express. 1986. 159 p.

3. La Fin des jeux, Montréal, Triptyque, 1986, 147 p. Prix Alfred-DesRochers, de l'Association des écrivains des Cantons de l'Est. 1986.

4. Voir la Fille à marier et le Péril amoureux que nous avons abordés dans deux chroniques précédentes. 